\title{
Reflets
}

Revue ontaroise d'intervention sociale et communautaire

\section{À la rencontre de deux solitudes}

\section{Myrtha Lapierre Peters}

Volume 4, numéro 1, printemps 1998

Intervention en contextes minoritaires

URI : https://id.erudit.org/iderudit/026211ar

DOI : https://doi.org/10.7202/026211ar

Aller au sommaire du numéro

Éditeur(s)

Reflets : Revue ontaroise d'intervention sociale et communautaire

ISSN

1203-4576 (imprimé)

1712-8498 (numérique)

Découvrir la revue

Citer cet article

Lapierre Peters, M. (1998). À la rencontre de deux solitudes. Reflets, 4(1),

217-224. https://doi.org/10.7202/026211ar

Tous droits réservés (C) Reflets : Revue ontaroise d'intervention sociale et communautaire, 1998

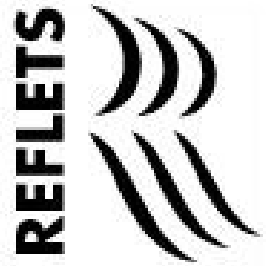

Ce document est protégé par la loi sur le droit d'auteur. L'utilisation des services d'Érudit (y compris la reproduction) est assujettie à sa politique d'utilisation que vous pouvez consulter en ligne.

https://apropos.erudit.org/fr/usagers/politique-dutilisation/ 


\title{
À la rencontre de deux solitudes
}

\author{
Myrtha Lapierre Peters
}

Consultante en formation et en développement communautaire, Ottawa

\section{Introduction et contexte}

La société canadienne est désormais pluriethnique et elle continuera de l'être car la mondialisation sera la réalité du $21^{\mathrm{e}}$ siècle.

"À l'exception des Amérindiens et des Inuits, l'histoire du peuplement canadien s'est façonnée à partir de l'immigration [...]. Alors qu'au dix-neuvième siècle, l'immigration avait été massivement britannique et française (...), depuis 1960 les immigrants d'origine non européenne constituent la majorité de l'ensemble des immigrants (...): les Caraïbéens, les Arabes, les Égyptiens, les Libanais, les Vietnamiens» (Barette et al. 1993:69-92).

Les Somaliens et d'autres ethnies se sont joints au groupe au cours de la dernière décennie. Cette pluriethnicité se manifeste dans toutes les sphères d'activité de cette société. Ainsi, sur le marché du travail, particulièrement dans le secteur de la santé, on retrouve un personnel provenant de l'immigration antillaise et africaine qui s'occupe des personnes âgées canadiennes de souche. Cette situation génère de nouvelles dimensions à la relation d'aide qui se trouve ainsi imprégnée de façons de faire ne correspondant pas toujours au fonctionnement antérieur et actuel des personnes âgées. Ainsi la communication et la réaction aux différents actes de la vie quotidienne se font parfois dans un labyrinthe. Ce manque 
de communication engendre deux solitudes : celle du personnel intervenant désigné «minorité raciale» et celle de sa clientèle âgée. Il y a lieu donc de se demander si une communication entre ces deux groupes est possible. Et dans le cas d'une réponse positive, cette communication peut-elle favoriser un rapprochement?

Outre le contexte créé par l'immigration, il faut aussi considérer les questions reliées à la culture et aux valeurs des deux groupes concernés. En effet, puisque la plupart des personnes âgées qui ont besoin d'intervention d'un personnel de «minorité raciale» avaient entre 30 et 50 ans quand les nouvelles cohortes de l'immigration non européenne sont arrivées, il y a eu peu de contact antérieur entre les personnes âgées actuelles et le personnel intervenant dérivant de cette nouvelle cohorte.

Ce peu de contact peut rendre difficile l'acceptation des différences perçues par les personnes âgées de souche, à cause du manque de sensibilisation aux différences culturelles et aux valeurs. Les différences culturelles se situent souvent dans le savoir, le savoirêtre, le savoir-faire, le savoir-agir, le savoir-interagir des groupes concernés et peuvent être source de choc ou tout simplement d'adaptation. Quant aux différences par rapport aux valeurs, elles sont liées «au processus de croissance et de maturation (...) et sont donc les plus difficiles à modifier» (Newman Giger et Davidhizar 1991: 190). Dans un tel contexte, les deux groupes présentent des différences culturelles fondamentales de par leur processus de croissance et de maturation dans des milieux différents. Toutefois, existe-t-il une possibilité de rapprochement qui transcendera les différences culturelles? C'est par le biais d'un sondage que nous avons voulu formuler des recommandations visant un rapprochement possible entre les solitudes des deux groupes, soit les personnes âgées et le personnel intervenant de «minorité raciale».

\section{Description de la démarche}

Dix participants bénévoles de chacun des deux groupes pré-cités ont été interviewés. Cette entrevue non structurée a été faite à 
l'aide d'un questionnaire qui portait sur les points suivants: les attitudes positives ou négatives des personnes âgées de souche envers le personnel intervenant désigné "minorité raciale»; certaines causes sous-jacentes à l'attitude positive ou négative des personnes âgées de souche envers le personnel intervenant désigné «minorité raciale»; la durée de l'attitude positive ou négative envers le personnel de «minorité raciale»; certains gains découlant de l'attitude positive des personnes âgées de souche; certaines pertes découlant de l'attitude négative envers le personnel intervenant de «minorité visible»; certaines solutions visant une découverte mutuelle et une démystification des barrières culturelles et raciales.

\section{Présentation et interprétation des données}

L'analyse des données recueillies permet de dégager des pistes par rapport aux questions posées et de mieux cerner certains éléments qui faciliteraient le rapprochement entre les deux groupes.

\section{Attitudes positives ou négatives des personnes âgées de souche envers le personnel intervenant désigné "minorité raciale»}

Les personnes âgées manifestent une attitude positive en s'exprimant ainsi: "Je vous remercie des bons soins». «Je suis contente que ce soit vous aujourd'hui». Elles ont tendance à remercier le personnel intervenant tantôt par des mots bienveillants tantôt par un sourire de gratification. Quant à l'attitude négative, elle devient évidente lorsque les personnes âgées demandent un intervenant autre qu'une personne de «minorité raciale». Parfois les personnes âgées posent directement des questions comme celles du genre: «Pourquoi cette personne doit-elle me frotter quand je ne suis pas sale ni dans une situation d'intimité»?

Pour le personnel intervenant, l'attitude positive des personnes âgées se reflète dans le soulagement qu'elles démontrent en accueillant le personnel intervenant. Les personnes âgées vont parfois jusqu’à leur offrir des petits cadeaux : «Voici un petit cadeau 
pour ta souplesse». L'attitude négative des personnes âgées est plutôt liée aux expressions ou au geste suivants : «Éloigne-toi de moi». «Je ne veux pas qu'une personne noire me touche, d'ailleurs vous autres me touchez trop". La personne âgée se tourne la tête ou couvre son visage.

Ainsi, comme on l'a constaté, ce sont des mots d'encouragement et de remerciement qui semblent le plus révéler une attitude positive entre les deux groupes. On remarque d'ailleurs que souvent le toucher prolongé est perçu par les personnes âgées comme un geste très intime alors que pour le personnel intervenant de «minorité raciale», c'est plutôt une marque d'affection, de délicatesse et de respect envers les personnes âgées. Cette différence culturelle peut être amoindrie dans la mesure où le personnel intervenant explique aux personnes âgées les raisons du toucher ou encore qu'il s'en abstient. Dans cet dernier cas, il peut toutefois les encourager en leur parlant.

\section{Certaines causes sous-jacentes à l'attitude positive ou négative de la personne âgée de souche}

Les personnes âgées se réferent à des contacts antérieurs tels un parent amérindien, des voisins de «minorité raciale» de leurs enfants pour expliquer leur attitude positive. Ces contacts antécédents leur ont permis de développer de bonnes perceptions des personnes différentes d'elles. Quant à l'attitude négative, les personnes âgées se réferent plutôt à la question économique, à savoir que «les étrangers nous font perdre nos emplois». Elles ne peuvent pas vérifier leurs dires mais ce sont des mythes qui sont répandus et elles y croient.

Le personnel intervenant trouve que l'attitude positive ou négative des personnes âgées est plutôt reliée au niveau d'éducation du bénéficiaire, à son degré d'ouverture d'esprit, à son histoire personnelle et familiale, à sa vision du rôle du personnel intervenant (serviteur ou personne aidante).

Ainsi, l'interprétation des causes sous-jacentes à l'attitude des personnes âgées semble varier beaucoup entre les deux groupes. Les personnes âgées qui ont eu des contacts antérieurs avec des 
gens de valeurs différentes semblent mieux préparées à avoir une attitude positive car elles ont dépassé le stade de choc et d'adaptation, tandis que celles qui n'ont pas été exposées à cette nouvelle vague d'immigration semblent percevoir leur présence comme une menace économique à la société du pays d'accueil. Le personnel intervenant semble plutôt utiliser des notions sociologiques pour trouver les causes de l'attitude positive ou négative.

\section{La durée de l'attitude positive ou négative selon les personnes âgées et selon le personnel intervenant}

Lorsque l'attitude des personnes âgées est positive, elle le demeure. Quant à l'attitude négative, elle peut durer quelques minutes ou quelques jours ou même perdurer. Cette situation prévaut dans les réponses des deux groupes.

Le personnel intervenant ajoute que les personnes âgées changent parfois leur attitude négative lorsqu'elles n'ont pas d'autre choix d'intervenant et doivent donc s'accommoder tant bien que mal de la situation.

\section{Certains gains découlant de l'attitude positive des personnes âgées de souche}

Selon les personnes âgées, une attitude positive engendre une meilleure relation, une collaboration accrue, un climat agréable, une communication plus facile. Voici un exemple de commentaire qui reflète les gains découlant de l'attitude positive des personnes âgées : «Je semble recevoir de bons soins».

En plus de rapporter les mêmes observations que les personnes âgées, le personnel intervenant ajoute: «Nous nous sentons plus utiles, nous avons plus de satisfaction personnelle, nous prêtons plus d'attention et il y a plus d'humanité pour les personnes âgées».

Les deux groupes semblent conscients des gains possibles dans une relation positive favorisant un rapprochement et évitant les deux solitudes. En effet, il semble que dès que la communication est bien établie, il y a possibilité de partager, de mieux exprimer ses besoins et de se faire comprendre mutuellement. 


\section{Certaines pertes découlant de l'attitude négative envers le personnel intervenant de «minorité raciale»}

L'attitude négative crée une relation de conflit d'après les personnes âgées. La communication semble difficile et il n'y a pas moyen d'établir une relation chaleureuse.

En plus de corroborer les observations des personnes âgées, celles du personnel intervenant reflètent un sentiment d'échec dans son rôle, un grand malaise, de la peine et du rejet.

Les deux groupes semblent donc conscients des effets néfastes d'une attitude négative. Les pertes éprouvées de part et d'autre se situent au niveau de la communication. D'ailleurs, le personnel intervenant, de par sa formation et du respect voué à son rôle de personne aidante, semble s'étonner de prime abord qu'une personne refuse ses interventions. Il peut même être dans un état de choc initial suivi d'une phase d'adaptation. La relation qui suit souffre d'un manque de dialogue ou de confiance. On doit alors se demander dans quelle mesure les interventions ne deviennent pas source de stress. D'où la nécessité de travailler dès le début à une amélioration de la communication.

\section{Certaines solutions si l'attitude est négative}

Au chapitre des solutions, les personnes âgées de souche ne se sont pas prononcées sur cette question quand elle a été posée.

Le personnel intervenant a formulé les propositions suivantes: «ré-offrir nos services, montrer notre côté humain, ne pas appliquer de pression, faire preuve de nos compétences professionnelles afin de créer un climat de confiance».

Le fait que les personnes âgées n'aient rien mentionné nous amène à poser certaines questions : Est-ce par ignorance des solutions, par manque d'affirmation, par peur de heurter l'autre ou par peur de se questionner? Les solutions proposées par le personnel intervenant semblent constructives. Elles permettent d'ouvrir le dialogue avec les personnes âgées et de faciliter le rapprochement par une perception positive au-delà des mythes et des stéréotypes. 


\section{Conclusion}

La pluriethnicité de la société canadienne apporte avec elle des défis quant aux relations qui vont se développer entre divers groupes : ceux de souche et ceux provenant de l'immigration non européenne. Dans le cas du personnel intervenant désigné «minorité raciale» et des personnes âgées de souche, ce défi passe par le rapprochement, bâti à travers une communication empreinte de confiance et de respect mutuel. En effet, même si l'échantillon était restreint, il y a lieu toutefois de considérer certaines pistes de réflexion s'inspirant des commentaires concernant les attitudes positives ou négatives des personnes âgées de souche, certaines causes sous-jacentes, la durée de l'attitude négative, les gains de l'attitude positive et les pertes associées à l'attitude négative. Ces pistes passent par l'aide et le support à fournir aux personnes âgées et au personnel intervenant de «minorité raciale» pour une meilleure compréhension de leurs différences culturelles. Le personnel intervenant offre d'ailleurs quelques solutions pour la création d'un climat de confiance.

De plus, pour le rapprochement de ces deux solitudes, nous recommandons au personnel intervenant certaines habiletés de communication interculturelle proposées par Barette (1993:144145), telles que :apprendre à se connaitre soi-même en explorant sa propre culture en plus de celle des autres et en tenant compte de l'influence de sa propre culture sur ses interprétations; éviter les jugements rapides en prenant le temps d'analyser une situation avant de sauter aux conclusions et en demandant des renseignements supplémentaires pour mieux comprendre; apprendre la négociation en cherchant les similitudes plutôt que les différences, en étant capable de se remettre en question et en cherchant le compromis qui permet de dépasser les différences; et finalement, être flexible et faire preuve de souplesse. 


\section{Bibliographie}

BARETTE Christian, Édithe GAUDET et Denyse LEMAY (1993). Guide de la communication interculturelle, Editions du Renouveau Pédagogique Inc., Montréal.

NEWMAN GIGER. Joyce et Ruth Elaine DAVIDHIZAR (1991). Soins infirmiers interculturels, Editeur Gaëtan Morin, Montréal. 